\title{
Response to: Alfred Sandrock, Wildon Farwell. Letter to the Editor, Comparisons Between Separately Conducted Clinical Trials: Letter to the Editor Regarding Dabbous O, Maru B, Jansen JP, Lorenzi M, Cloutier M, Guérin A, et al. Adv Ther (2019) 36(5):1164-76. doi:10.1007/s12325-019-00923-8
}

Omar Dabbous · Benit Maru · Jeroen P. Jansen · Maria Lorenzi · Martin Cloutier •

Annie Guérin · Irina Pivneva · Eric Q. Wu · Ramesh Arjunji · Douglas Feltner · Douglas M. Sproule

Received: August 27, 2019 / Published online: September 11, 2019

(C) The Author(s) 2019

Keywords: AVXS-101; Indirect treatment comparison; Nusinersen; Neuroscience; Onasemnogene abeparvovec; Spinal muscular atrophy type 1 (SMA type 1 )

We thank Sandrock and Farwell for their interest in our study and thank them for highlighting several of the strengths and limitations of the work discussed in this article, which attempts to offer a perspective on a repeated question (comparability of therapeutic options) posed by clinicians and health authorities.

Spinal muscular atrophy (SMA) type 1 is a rare and severe disease with a universally dismal prognosis for patients, as demonstrated in multiple natural history studies [1-3]. Multiple

O. Dabbous · B. Maru · R. Arjunji · D. Feltner .

D. M. Sproule $(\varangle)$

AveXis, Inc, Bannockburn, IL, USA

e-mail: dsproule216@avexis.com

O. Dabbous

e-mail: odabbous902@avexis.com

J. P. Jansen · M. Lorenzi

Precision Xtract, Oakland, CA, USA

M. Cloutier · A. Guérin · I. Pivneva

Analysis Group, Inc, Montreal, QC, Canada

E. Q. Wu

Analysis Group, Inc, Boston, MA, USA natural history studies have established that patients with the classic SMA type 1 phenotype and genotype ( 2 copies of the survival motor neuron 2 gene) experience predictably poor survival, universally decline in motor function after disease onset, and gain no milestones without therapeutic treatment [1-5]. These findings were re-affirmed by the universally dismal course seen in the sham (control) arm of the ENDEAR study [6]. As such, an open-label, single-arm trial design is reasonable, ethical, and appropriate in this ultra-rare patient population, particularly given the magnitude of the therapeutic impact seen with both therapies (rendering the assignment of control or drug obvious to the trial clinicians, despite the "blind"). Indeed, similar survival and motor function were observed in the open-label, dose escalation study (CS3A) [7] and sham-controlled ENDEAR (CS3B) studies of nusinersen [6], emphasizing the comparability of open-label and blinded studies in SMA type 1.

The points raised by Sandrock and Farwell regarding the limitations of the methodology used in our study and the differences between the ENDEAR and CL-101 trials are reasonable and thus acknowledged and extensively discussed in our article. Regarding the use of the number needed to treat (NNT) analysis, we concur that NNT is a measure ideally used within a specific trial and agree that valid 
comparisons of two treatments in separate trials are contingent on close similarity of trial populations, outcome measures, and other trial characteristics. However, in the absence of head-to-head trials, a between-trial comparison of the reported results of the two trials is an appropriate method to compare the relative treatment effects of nusinersen and AVXS-101 (onasemnogene abeparvovec). We consider that patient baseline characteristics in the ENDEAR and CL-101 trials do not differ to such an extent as to invalidate their comparison using NNT.

While important differences do exist between study populations, these differences and the impact on the potential for therapeutic response to therapy are not as stark as characterized in the letter. The authors highlight differences in patient baseline characteristics between the two trials regarding (1) age, (2) disease duration, (3) mean motor function, and (4) disease burden. Regarding age and disease duration, some infants in ENDEAR were diagnosed and treated later, on average, than those in CL-101 (mean age at first dose 3.4 vs. 5.4 months). We have acknowledged this limitation; it is important, however, to note the broad overlap in the distribution of age between the CL-101 (range: 0.9-7.9 months) and ENDEAR (range: 1.7-7.9 months) trials. To the extent that the age distribution of patients in ENDEAR could be replicated within the CL-101 by, for example, reweighing the patients based on their age at treatment initiation to replicate the age distribution of patients in ENDEAR, or excluding the patients treated at an early age (e.g., below the age of 1.7 months in CL-101), the outcomes for onasemnogene abeparvovec for event-free survival and Children's Hospital of Philadelphia Infant Test of Neuromuscular Disorders [(CHOPINTEND); increase of at least 4 points] would remain at $100 \%$, despite age adjustments as all patients achieved these outcomes in the CL-101 trial. Even for motor-milestone achievements not reached by $100 \%$ of patients in CL-101, the differences between the two trials are clearly evident (e.g., sit unassisted: 92\% in CL-101 vs. $8 \%$ in ENDEAR) so that implementing reweighing techniques or excluding patients below the age of 1.7 months would probably not impact the study results and conclusions. In addition, although some infants in the CL-101 trial received their trial dose in the first few months of life, half (3/6) of these children already had CHOP-INTEND scores $<20$ at that early point, indicative of severe, early disease onset and poor motor function within the first month of life (SMA type 1a), even by the standards of this disease. Such patients with extremely active disease (poor motor function and impaired swallowing) before 1 month of age have very poor prognosis among patients with SMA and would not be expected to survive beyond a few months of age without intervention [4]. Therefore, younger age at first dose, per se, may not necessarily be associated with more favorable outcomes in CL-101 patients, as suggested by Sandrock and Farwell.

With respect to motor function score at baseline, Sandrock and Farwell state that CHOPINTEND scores in ENDEAR were lower than those of CL-101, suggesting that ENDEAR patients were weaker at baseline. The mean baseline CHOP-INTEND score was 26.6 in ENDEAR and 28 in CL-101, which are not materially different.

Regarding disease burden at baseline, Sandrock and Farwell speculate that outcomes between the two trials are likely influenced by a greater disease burden of nusinersen-treated patients, notably a higher proportion of patients dependent on respiratory support and having swallowing difficulties. Without access to individual-level data from ENDEAR, whether or not differences in ventilatory support at baseline relate to permanent assisted ventilation during the study period remains speculative. However, we do observe that $17 \%$ of patients treated with onasemnogene abeparvovec had ventilatory use at baseline and $0 \%$ required permanent assisted ventilation during the study period, whereas $26 \%$ of patients treated with nusinersen had ventilatory use at baseline and 22\% required permanent assisted ventilation during the study period. We disagree with the assertion that the entry criteria related to nutritional support differed materially between these studies. Provision of nutritional support in infants in CL-101 with poor swallowing function (5 infants- $42 \%$ required enteral feeding at baseline, a higher proportion than reported in ENDEAR) was intended to ensure 
adequate nutrition and hydration, consistent with established standards of care $[8,9]$. A similar eligibility criterion, and expectation that study participants would receive basic standard of care, was applied in the ENDEAR trial: "infants receiving adequate nutrition and hydration (with or without gastrostomy) in the opinion of the site investigator at the time of study entry" as well as the requirement that participants measure at least the third percentile in body weight [6]. We agree with the critique that clinical trials, in general, are not designed to represent a wide real-world population and often have narrow eligibility criteria. In this regard, both the ENDEAR and CL-101 trials may raise generalizability questions and may not fully reflect the wider population of patients with SMA type 1.

Sandrock and Farwell specifically question the inclusion of two subjects in the CL-101 study who had baseline scores above the range expected for symptomatic type 1 patients. We agree that these two patients, identified through astute clinical care and treated when early symptoms of disease were present, provided a greater opportunity for clinical response; unmentioned by Sandrock and Farwell is that several patients in the ENDEAR study were enrolled with similar baseline motor function (CHOP-INTEND scores above 40, and even approaching 50), though without a similar clinical response [10, 11].

As Sandrock and Farwell rightly point out, head-to-head clinical trials are the gold standard for comparative effectiveness but are seldom performed. However, our study is based on the best available evidence, and while the results of the study should be interpreted in light of the limitations, it provides stakeholders with additional tools to inform their decisions. Further comparison of outcomes is warranted as additional data on the efficacy and safety of the onasemnogene abeparvovec and nusinersen become available.

\section{ACKNOWLEDGEMENTS}

Funding. There was no funding for the publication of this letter.
Authorship. All named authors meet the International Committee of Medical Journal Editors (ICMJE) criteria for authorship for this article, take responsibility for the integrity of the work as a whole, and gave their approval for this version to be published.

Disclosures. Benit Maru is an employee of SSI Strategy, who are contracted to support AveXis, Inc. Jeroen P. Jansen is an employee of Precision Xtract, which received consultancy fees from AveXis, Inc., for this work. Maria Lorenzi is an employee of Precision Xtract, which received consultancy fees from AveXis, Inc., for this work. Omar Dabbous is an employee of AveXis. Ramesh Arjunji is an employee of AveXis. Douglas Feltner is an employee of AveXis. Douglas M. Sproule is an employee of AveXis. Martin Cloutier is an employee of Analysis Group, Inc., which received consultancy fees from AveXis for this work. Annie Guérin is an employee of Analysis Group, Inc., which received consultancy fees from AveXis for this work. Irina Pivneva is an employee of Analysis Group, Inc., which received consultancy fees from AveXis for this work. Eric Q. Wu is an employee of Analysis Group, Inc., which received consultancy fees from AveXis for this work.

Compliance with Ethics Guidelines. This letter is a response to the letter to the editor on the article that was based on previously conducted studies and did not contain any studies with human participants or animals performed by any of the authors.

Open Access. This article is distributed under the terms of the Creative Commons Attribution-NonCommercial 4.0 International License (http://creativecommons.org/licenses/ by-nc/4.0/), which permits any noncommercial use, distribution, and reproduction in any medium, provided you give appropriate credit to the original author(s) and the source, provide a link to the Creative Commons license, and indicate if changes were made. 


\section{REFERENCES}

1. Finkel RS, McDermott MP, Kaufmann P, et al. Observational study of spinal muscular atrophy type I and implications for clinical trials. Neurology. 2014;83(9):810-7.

2. Wadman RI, Stam M, Gijzen M, et al. Association of motor milestones, SMN2 copy and outcome in spinal muscular atrophy types $0-4$. J Neurol Neurosurg Psychiatry. 2017;88(4):365-7.

3. Kolb SJ, Coffey CS, Yankey JW, et al. Natural history of infantile-onset spinal muscular atrophy. Ann Neurol. 2017;82(6):883-91.

4. De Sanctis R, Pane M, Coratti G, et al. Clinical phenotypes and trajectories of disease progression in type 1 spinal muscular atrophy. Neuromuscul Disord. 2018;28(1):24-8.

5. De Sanctis R, Coratti G, Pasternak A, et al. Developmental milestones in type I spinal muscular atrophy. Neuromuscul Disord. 2016;26(11):754-9.

6. Finkel RS, Mercuri E, Darras BT, et al. Nusinersen versus sham control in infantile-onset spinal muscular atrophy. N Engl J Med. 2017;377(18):1723-32.
7. Finkel RS, Chiriboga CA, Vajsar J, et al. Treatment of infantile-onset spinal muscular atrophy with nusinersen: a phase 2, open-label, dose-escalation study. Lancet. 2016;388(10063):3017-26.

8. Mercuri E, Finkel RS, Muntoni F, et al. Diagnosis and management of spinal muscular atrophy: part 1: recommendations for diagnosis, rehabilitation, orthopedic and nutritional care. Neuromuscul Disord. 2018;28(2):103-15.

9. Wang $\mathrm{CH}$, Finkel RS, Bertini ES, et al. Consensus statement for standard of care in spinal muscular atrophy. J Child Neurol. 2007;22(8):1027-49.

10. Finkel RS, Mercuri E, Darras BT, et al. Supplementay Appendix. Nusinersen versus Sham Control in Infantile-Onset Spinal Muscular Atrophy. 2017. https://www.nejm.org/doi/suppl/10.1056/NEJMoa170 2752/suppl_file/nejmoa1702752_appendix.pdf. Accessed 20 Aug 2019.

11. Mendell JR, Al-Zaidy S, Shell R, et al. Single-dose gene-replacement therapy for spinal muscular atrophy. N Engl J Med. 2017;377(18):1713-22. 